Supporting Information for:

\title{
Characterization of Porphyrin Surface Orientation in Monolayers on Au(111) and Si(100) using Spectroscopically Labeled Molecules
}

\author{
Jieying Jiao, Patchanita Thamyongkit, Izabela Schmidt, \\ Jonathan S. Lindsey, and David F. Bocian
}

\section{Table of Contents}

\section{Experimental Section for Synthesis Procedures $\quad \mathrm{S} 1-\mathrm{S} 7$}

1. General Procedures. All ${ }^{1} \mathrm{H}$ NMR spectra $(300 \mathrm{MHz})$ and ${ }^{13} \mathrm{C}$ NMR $(75 \mathrm{MHz})$ were collected in $\mathrm{CDCl}_{3}$ unless noted otherwise. Absorption and fluorescence spectra were collected in toluene at room temperature unless noted otherwise. Mass spectra of porphyrins were obtained via laser desorption mass spectrometry (LD-MS) without a matrix ${ }^{31}$ and by highresolution fast atom bombardment mass spectrometry (FAB-MS) using a matrix of nitrobenzyl alcohol and polyethylene glycol. Melting points are uncorrected. Silica gel $(40 \mu \mathrm{m}$ average particle size) was used for column chromatography. THF was freshly distilled from sodium as required. Toluene was distilled from $\mathrm{CaH}_{2} . \mathrm{CHCl}_{3}$ was stabilized with $0.8 \%$ ethanol.

2. Noncommercial compounds. Isotopically labeled compounds pyrrole- $d_{5}{ }^{18}$ and $p$ tolualdehyde-methyl- $d_{3} ;{ }^{20}$ dipyrromethanes $\mathbf{1 b},{ }^{22} \quad \mathbf{1 c},,^{21} \quad \mathbf{1 e},^{23}$ and $\mathbf{1 f} ;{ }^{5 \mathrm{~d}}$ and $1,9-$ diacyldipyrromethanes $\mathbf{4}^{27}$ and $\mathbf{5 - S n B u _ { 2 }}{ }^{29}$ were obtained by literature procedures.

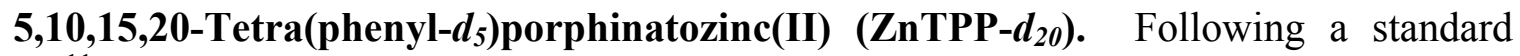
procedure, ${ }^{11}$ a mixture of pyrrole $(55.0 \mu \mathrm{L}, 0.787 \mathrm{mmol})$, benzaldehyde- $d_{5}(80.0 \mu \mathrm{L}, 0.787$ mmol) in $\mathrm{CH}_{2} \mathrm{Cl}_{2}(79 \mathrm{~mL})$ was degassed for $5 \mathrm{~min}$ and then treated with $\mathrm{BF}_{3} \cdot \mathrm{O}(\mathrm{Et})_{2}(3.20 \mu \mathrm{L}$, $0.025 \mathrm{mmol})$ and TFA $(91.0 \mu \mathrm{L}, 1.18 \mathrm{mmol})$ at once at room temperature. After $1 \mathrm{~h}$, DDQ (135 $\mathrm{mg}, 0.595 \mathrm{mmol})$ was added. The reaction mixture was stirred for $1 \mathrm{~h}$ and then TEA $(0.50 \mathrm{~mL})$ was added. The mixture was passed through a silica pad $\left(\mathrm{CH}_{2} \mathrm{Cl}_{2}\right)$ and the fractions were collected until the eluate was no longer dark. After removal of the solvent, the resulting crude product was dissolved in $\mathrm{CHCl}_{3}(50 \mathrm{~mL})$ and then treated overnight with a solution of $\mathrm{Zn}(\mathrm{OAc})_{2} \cdot 2 \mathrm{H}_{2} \mathrm{O}(216 \mathrm{mg}, 0.984 \mathrm{mmol})$ in methanol $(12 \mathrm{~mL})$ at room temperature. The reaction mixture was washed with water, dried $\left(\mathrm{Na}_{2} \mathrm{SO}_{4}\right)$ and concentrated to dryness. Purification by column chromatography [silica, $\mathrm{CH}_{2} \mathrm{Cl}_{2}$ /hexanes (2:1)] afforded a purple solid $(74 \mathrm{mg}, 54 \%):{ }^{1} \mathrm{H}$ NMR $\delta 8.92(\mathrm{~s}, 8 \mathrm{H}) ;{ }^{13} \mathrm{C}$ NMR $\delta 120.7,125.5,125.8,126.1,131.8$ (enh), 133.4, 133.8, 134.1,

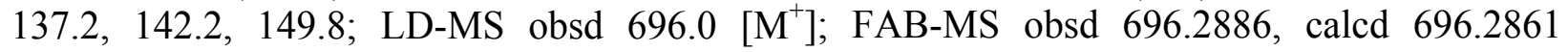
$\left(\mathrm{C}_{44} \mathrm{H}_{45} \mathrm{D}_{20} \mathrm{~N}_{4} \mathrm{Zn}\right) ; \lambda_{\text {abs }} 423,549 \mathrm{~nm} ; \lambda_{\text {em }}\left(\lambda_{\text {ex }}=550 \mathrm{~nm}\right) 596,646 \mathrm{~nm}$.

Mesitaldehyde-3,5- $\boldsymbol{d}_{2}$. A sample of mesitaldehyde $(0.500 \mathrm{~g}, 3.37 \mathrm{mmol})$ was slowly treated with $\mathrm{D}_{2} \mathrm{SO}_{4}(6.75 \mathrm{~g}, 67.5 \mathrm{mmol})$ at room temperature. The mixture was heated to $65{ }^{\circ} \mathrm{C}$ and stirred for $2 \mathrm{~h}$. After cooling to room temperature, the mixture was treated dropwise with $\mathrm{D}_{2} \mathrm{O}(1 \mathrm{~mL})$ and rinsed with ice water $(10 \mathrm{~mL})$ and $\mathrm{CH}_{2} \mathrm{Cl}_{2}(10 \mathrm{~mL})$. The organic phase was concentrated and dried $\left(\mathrm{Na}_{2} \mathrm{SO}_{4}\right)$. The procedure was repeated twice. The crude mixture was purified by column chromatography [silica, hexanes $/ \mathrm{CH}_{2} \mathrm{Cl}_{2}(2: 1)$ ] to afford a pale yellow oil with $>97 \%$ isotopic purity $(0.350 \mathrm{~g}):{ }^{1} \mathrm{H}$ NMR $\delta 2.26(\mathrm{~s}, 3 \mathrm{H}), 2.51(\mathrm{~s}, 6 \mathrm{H}), 10.48(\mathrm{~s}, 1 \mathrm{H}) ;{ }^{13} \mathrm{C}$ NMR $\delta 20.0,20.9,129.5,129.6,129.8,130.2,141.0,143.2,192.3$ (enh). 
5,10,15,20-Tetra(mesityl-3,5- $\boldsymbol{d}_{2}$ )porphinatozinc(II) (ZnTMP-Ar- $\boldsymbol{d}_{\boldsymbol{8}}$ ). Following a standard procedure, ${ }^{15}$ a mixture of pyrrole $(116 \mu \mathrm{L}, 1.67 \mathrm{mmol})$ and mesitaldehyde- $3,5-d_{2}(250$ $\mathrm{mg}, 1.66 \mathrm{mmol})$ in $\mathrm{CHCl}_{3}(166 \mathrm{~mL})$ was degassed for $5 \mathrm{~min}$ and then treated with $\mathrm{BF}_{3} \cdot \mathrm{O}(\mathrm{Et})_{2}$ $(69.5 \mu \mathrm{L}, 0.548 \mathrm{mmol})$ at room temperature. After $1 \mathrm{~h}$, DDQ $(283 \mathrm{mg}, 1.25 \mathrm{mmol})$ was added. The reaction mixture was stirred for $1 \mathrm{~h}$ and then TEA $(0.50 \mathrm{~mL})$ was added. The mixture was filtered through a silica pad $\left(\mathrm{CH}_{2} \mathrm{Cl}_{2}\right)$, and fractions were collected until the eluate was no longer dark. After removal of the solvent, the resulting crude product was dissolved in $\mathrm{CHCl}_{3}(100 \mathrm{~mL})$ and then treated overnight with a solution of $\mathrm{Zn}(\mathrm{OAc})_{2} \cdot 2 \mathrm{H}_{2} \mathrm{O}(457 \mathrm{mg}, 2.08 \mathrm{mmol})$ in methanol $(25 \mathrm{~mL})$ at room temperature. The reaction mixture was washed with water, dried $\left(\mathrm{Na}_{2} \mathrm{SO}_{4}\right)$ and concentrated to dryness. Purification by column chromatography [silica, $\mathrm{CH}_{2} \mathrm{Cl}_{2} /$ hexanes $(2: 1)$ ] afforded a purple solid $(90 \mathrm{mg}, 25 \%):{ }^{1} \mathrm{H}$ NMR $\left(\mathrm{CS}_{2}\right) \delta 2.11(\mathrm{~s}, 24 \mathrm{H}), 2.88(\mathrm{~s}, 12 \mathrm{H}), 8.85(\mathrm{~s}$, $8 \mathrm{H})$; a ${ }^{13} \mathrm{C}$ NMR spectrum could not be obtained due to low solubility; LD-MS obsd $852.0\left[\mathrm{M}^{+}\right]$; FAB-MS obsd 852.4000, calcd $852.3986\left(\mathrm{C}_{56} \mathrm{H}_{44} \mathrm{D}_{8} \mathrm{~N}_{4} \mathrm{Zn}\right) ; \lambda_{\text {abs }} 421,549 \mathrm{~nm} ; \lambda_{\text {em }}\left(\lambda_{\text {ex }}=550 \mathrm{~nm}\right)$ $591,644 \mathrm{~nm}$.

$\mathbf{5 , 1 0 , 1 5 , 2 0 - T e t r a m e s i t y l p o r p h i n a t o z i n c ( I I ) - ~} \beta-d_{s}\left(\mathrm{ZnTMP}-\beta-d_{g}\right)$. A mixture of pyrrole$d_{5}(120 \mu \mathrm{L}, 1.66 \mathrm{mmol})$ and mesitaldehyde $(247 \mathrm{mg}, 1.67 \mathrm{mmol})$ in $\mathrm{CH}_{2} \mathrm{Cl}_{2}(165 \mathrm{~mL})$ was degassed for $5 \mathrm{~min}$ and then treated with $\mathrm{BF}_{3} \cdot \mathrm{O}(\mathrm{Et})_{2}(69.5 \mu \mathrm{L}, 0.548 \mathrm{mmol})$ and ethanol- $d_{l}(1.24$ $\mathrm{mL}, 0.75 \%)$ at room temperature. After $1 \mathrm{~h}$, DDQ $(283 \mathrm{mg}, 1.25 \mathrm{mmol})$ was added. The reaction mixture was stirred for $1 \mathrm{~h}$ and then TEA $(0.50 \mathrm{~mL})$ was added. The mixture was filtered through a silica pad $\left(\mathrm{CH}_{2} \mathrm{Cl}_{2}\right)$, and fractions were collected until the eluate was no longer dark. After removal of the solvent, the resulting crude product was dissolved in $\mathrm{CH}_{2} \mathrm{Cl}_{2}(50 \mathrm{~mL})$ and then treated overnight with a solution of $\mathrm{Zn}(\mathrm{OAc})_{2}(382 \mathrm{mg}, 2.08 \mathrm{mmol})$ in $\mathrm{CD}_{3} \mathrm{OD} / \mathrm{D}_{2} \mathrm{O}$ (4 $\mathrm{mL}, 20: 1)$ at room temperature. TLC analysis exhibited residual free base product. Hence, the reaction mixture was washed with water and the zinc metalation was repeated for $4 \mathrm{~h}$. After washing with water, the reaction mixture was dried $\left(\mathrm{Na}_{2} \mathrm{SO}_{4}\right)$ and concentrated to dryness. Purification by column chromatography [silica, $\mathrm{CH}_{2} \mathrm{Cl}_{2} /$ hexanes (2:1)] afforded a purple solid (90 $\mathrm{mg}, 83 \%$ isotopically pure).

5-[p-Tolyl $\left(\right.$ methyl- $\left.\left.\boldsymbol{d}_{3}\right)\right]$ dipyrromethane (1a). Following a standard procedure, ${ }^{19}$ a mixture of $p$-tolualdehyde-methyl- $d_{3}(0.500 \mathrm{~g}, 4.06 \mathrm{mmol})$ and pyrrole $(28.0 \mathrm{~mL}, 404 \mathrm{mmol})$ was degassed for $5 \mathrm{~min}$ and treated with $\mathrm{InCl}_{3}(0.090 \mathrm{~g}, 0.407 \mathrm{mmol})$ at room temperature. After stirring for $1.5 \mathrm{~h}$, powdered $\mathrm{NaOH}(4.87 \mathrm{~g}, 122 \mathrm{mmol})$ was added and the mixture was stirred for $45 \mathrm{~min}$. The reaction mixture was filtered, and the filtrated was concentrated to dryness. Recrystallization of the resulting brown crude mixture $(\mathrm{MeOH} /$ water) gave a colorless solid (0.652 g, 67\%): mp $113{ }^{\circ} \mathrm{C} ;{ }^{1} \mathrm{H}$ NMR $5.45(\mathrm{~s}, 1 \mathrm{H}), 5.88-5.98(\mathrm{~m}, 2 \mathrm{H}), 6.14-6.24(\mathrm{~m}, 2 \mathrm{H}), 6.70$ $6.72(\mathrm{~m}, 2 \mathrm{H}), 7.10(\mathrm{~d}, J=8.4 \mathrm{~Hz}, 1 \mathrm{H}), 7.13(\mathrm{~s}, 2 \mathrm{H}), 7.45(\mathrm{~d}, J=8.4 \mathrm{~Hz}, 1 \mathrm{H}), 7.94(\mathrm{brs}, 2 \mathrm{H}) ;{ }^{13} \mathrm{C}$ NMR $\delta$ 43.6, 107.1, 107.4, 108.4, 108.5, 117.1, 117.5, 120.8, 128.2, 129.3, 130.1, 131.7, 132.7, 139.0, 141.2; FAB-MS obsd 239.1497, calcd $239.1502\left(\mathrm{C}_{16} \mathrm{H}_{13} \mathrm{D}_{3} \mathrm{~N}_{2}\right)$.

5-(4-(Trifluoromethyl)phenyl)dipyrromethane (1b). Following a standard procedure, ${ }^{19}$ a mixture of 4-(trifluoromethyl)benzaldehyde $(1.00 \mathrm{~g}, 5.74 \mathrm{mmol})$ and pyrrole $(40.0$ $\mathrm{mL}, 577 \mathrm{mmol})$ was degassed for $5 \mathrm{~min}$ and treated with $\mathrm{InCl}_{3}(0.127 \mathrm{~g}, 0.574 \mathrm{mmol})$ at room temperature. After stirring for $1.5 \mathrm{~h}$, powdered $\mathrm{NaOH}(6.90 \mathrm{~g}, 173 \mathrm{mmol})$ was added, and the mixture was stirred for $45 \mathrm{~min}$. The reaction mixture was filtered, and the filtrate was concentrated to dryness. Recrystallization of the resulting brown crude mixture (EtOH/water) gave a colorless solid $(1.15 \mathrm{~g}, 69 \%): \mathrm{mp} 102-103{ }^{\circ} \mathrm{C} ;{ }^{1} \mathrm{H}$ NMR $\delta 5.54(\mathrm{~s}, 1 \mathrm{H}), 5.88-5.93(\mathrm{~m}$, $2 \mathrm{H}), 6.19(\mathrm{dd}, J=6.0 \mathrm{~Hz}, J=3.0 \mathrm{~Hz}, 2 \mathrm{H}), 6.763(\mathrm{dd}, J=6.0 \mathrm{~Hz}, J=3.0 \mathrm{~Hz}, 2 \mathrm{H}), 7.34(\mathrm{~d}, J=$ $8.1 \mathrm{~Hz}, 2 \mathrm{H}), 7.59(\mathrm{~d}, J=8.4 \mathrm{~Hz}, 2 \mathrm{H}), 7.94(\mathrm{brs}, 2 \mathrm{H}) ;{ }^{13} \mathrm{C}$ NMR $\delta 43.8,107.6,108.6,117.7$, 
125.48, 125.53, 128.7, 131.5, 146.2; Anal Calcd for $\mathrm{C}_{16} \mathrm{H}_{13} \mathrm{~F}_{3} \mathrm{~N}_{2}$ : C, 66.20; H, 4.51; N, 9.65; Found: C, 66.15; H, 4.51; N, 9.66.

Dibutyl[5,10-dihydro-1,9-di-p-toluoyl-5-p-tolyl(methyl-d d $\left._{3}\right)$ dipyrrinato]tin(IV)

$\left.\mathbf{S n B u}_{2}\right)$. Following a standard procedure, ${ }^{24}$ a solution of $1 \mathbf{a}(500 \mathrm{mg}, 2.09 \mathrm{mmol})$ and $p$-toluoyl chloride $(553 \mu \mathrm{L}, 4.18 \mathrm{mmol})$ in 1,2-dichloroethane $(42 \mathrm{~mL})$ was treated with $\mathrm{SbCl}_{5}(535 \mu \mathrm{L}$, $4.19 \mathrm{mmol})$ at room temperature. After $5 \mathrm{~min}$, water $(50 \mathrm{~mL})$ was added. The reaction mixture was stirred for $1 \mathrm{~min}$ and poured into saturated aqueous $\mathrm{NaHCO}_{3}$. After vigorously stirring for $30 \mathrm{~min}$, the organic layer was separated, dried $\left(\mathrm{Na}_{2} \mathrm{SO}_{4}\right)$ and concentrated to dryness. The resulting crude product was dissolved in $\mathrm{CH}_{2} \mathrm{Cl}_{2}(4 \mathrm{~mL})$ and the mixture was treated with TEA $(871 \mu \mathrm{L}, 6.27 \mathrm{mmol})$ and $\mathrm{Bu}_{2} \mathrm{SnCl}_{2}(635 \mathrm{mg}, 2.09 \mathrm{mmol})$ at room temperature. TLC analysis $\left(\mathrm{CH}_{2} \mathrm{Cl}_{2}\right.$ /ethyl acetate 95:5) showed the reaction to be complete after $30 \mathrm{~min}$. The solvent was removed and the residue was purified by flash column chromatography $\left(\mathrm{CH}_{2} \mathrm{Cl}_{2}\right)$, obtaining a green solid as the first band. Recrystallization (diethyl ether/MeOH) gave a pale yellow solid (0.484 g, 33\%): mp $128-129{ }^{\circ} \mathrm{C}$ (dec.); ${ }^{1} \mathrm{H}$ NMR (400 MHz) $\delta 0.67-0.77(\mathrm{~m}, 6 \mathrm{H}), 1.09-1.71$ (m, 12H), $2.45(\mathrm{~s}, 6 \mathrm{H}), 5.57(\mathrm{~s}, 1 \mathrm{H}), 6.20(\mathrm{~d}, J=4.0 \mathrm{~Hz}, 2 \mathrm{H}), 7.09-7.12(\mathrm{~m}, 6 \mathrm{H}), 7.31(\mathrm{~d}, J=8.0 \mathrm{~Hz}$, 4H), $7.83(\mathrm{~d}, J=8.0 \mathrm{~Hz}, 4 \mathrm{H}) ;{ }^{13} \mathrm{C} \mathrm{NMR}(100 \mathrm{MHz}) \delta 13.6,21.6,23.9,24.7,25.9,26.3,27.17$, 27.23, 45.3, 115.0, 123.7, 127.9, 128.6, 129.0, 129.1, 129.3, 131.7, 135.0, 135.7, 141.3, 142.1, 151.7, 184.3; FAB-MS obsd 707.2659, calcd 707.2613 $\left(\mathrm{C}_{40} \mathrm{H}_{41} \mathrm{D}_{3} \mathrm{~N}_{2} \mathrm{O}_{2} \mathrm{Sn}\right)$.

Dibutyl[5,10-dihydro-1,9-di-p-toluoyl-5-(4-trifluoromethyl)phenyl)dipyrrinato]$\operatorname{tin}(\mathbf{I V})\left(\mathbf{3 - S n B u _ { 2 }}\right)$. Following a standard procedure, ${ }^{24}$ a solution of $\mathbf{1 b}(800 \mathrm{mg}, 2.76 \mathrm{mmol})$ and p-toluoyl chloride $(729 \mu \mathrm{L}, 5.51 \mathrm{mmol})$ in 1,2-dichloroethane $(55 \mathrm{~mL})$ was treated with $\mathrm{SbCl}_{5}$ $(706 \mu \mathrm{L}, 4.19 \mathrm{mmol})$ at room temperature for $5 \mathrm{~min}$. Water $(50 \mathrm{~mL})$ was added and the reaction mixture was stirred for $1 \mathrm{~min}$. The mixture was poured into saturated aqueous $\mathrm{NaHCO}_{3}$ and vigorously stirred for $30 \mathrm{~min}$. The organic layer was separated, dried $\left(\mathrm{Na}_{2} \mathrm{SO}_{4}\right)$ and concentrated to dryness. The resulting crude product was dissolved in $\mathrm{CH}_{2} \mathrm{Cl}_{2}(5.5 \mathrm{~mL})$ and the mixture was treated with TEA $(1.15 \mathrm{~mL}, 8.27 \mathrm{mmol})$ and $\mathrm{Bu}_{2} \mathrm{SnCl}_{2}(837 \mathrm{mg}, 2.76 \mathrm{mmol})$ at room temperature. TLC analysis $\left(\mathrm{CH}_{2} \mathrm{Cl}_{2}\right.$ /ethyl acetate 95:5) showed the reaction to be complete after $30 \mathrm{~min}$. The solvent was removed and the residue was purified by flash column chromatography $\left(\mathrm{CH}_{2} \mathrm{Cl}_{2}\right)$, obtaining a dark green solid as the first band. Recrystallization (diethyl ether/MeOH) gave a pale yellow solid (1.04 g, 50\%): mp 143-144 ${ }^{\circ} \mathrm{C}$ (dec.); ${ }^{1} \mathrm{H}$ NMR $\delta 0.67-0.77$ (m, 6H), $1.06-1.73(\mathrm{~m}, 12 \mathrm{H}), 2.46(\mathrm{~s}, 6 \mathrm{H}), 5.67(\mathrm{~s}, 1 \mathrm{H}), 6.22(\mathrm{~d}, J=3.6 \mathrm{~Hz}, 2 \mathrm{H}), 7.12(\mathrm{~d}, J=3.9 \mathrm{~Hz}, 2 \mathrm{H})$, $7.31-7.35(\mathrm{~m}, 6 \mathrm{H}), 7.55(\mathrm{~d}, J=8.1 \mathrm{~Hz}, 2 \mathrm{H}), 7.85(\mathrm{~d}, J=8.1 \mathrm{~Hz}, 4 \mathrm{H}) ;{ }^{13} \mathrm{C} \mathrm{NMR} \delta 13.5,21.6$, 23.9, 24.9, 25.9, 26.3, 27.2, 27.3, 45.3, 115.0, 123.6, 125.5, 125.6, 128.4, 129.1, 129.2, 134.8, 136.1, 142.3, 148.3, 150.0, 184.7; Anal Calcd for $\mathrm{C}_{40} \mathrm{H}_{41} \mathrm{~F}_{3} \mathrm{~N}_{2} \mathrm{O}_{2} \mathrm{Sn}$ : C, 63.43; H, 5.46; N, 3.70; Found: C, 63.50; H, 5.42; N, 3.73.

15-(4-(S-Acetylthiomethyl)phenyl)-5-p-tolyl(methyl-d $\left.d_{3}\right)-10,20-d i-p$-tolylporphinatozinc(II) (ZnP-D $\left.{ }^{\mathbf{D}} \mathbf{B z S A c}\right)$. Following a standard procedure ${ }^{26}$ under improved conditions, ${ }^{24,25}$ a solution of 2-SnBu 2 (296 mg, $0.419 \mathrm{mmol})$ in dry THF/MeOH (17 mL, 10:1) was treated with $\mathrm{NaBH}_{4}(634 \mathrm{mg}, 16.7 \mathrm{mmol})$ under argon at room temperature for $4 \mathrm{~h}$. The reaction mixture was poured into a mixture of saturated aqueous $\mathrm{NH}_{4} \mathrm{Cl}$ and $\mathrm{CH}_{2} \mathrm{Cl}_{2}$. The organic phase was separated, washed with water, dried $\left(\mathrm{Na}_{2} \mathrm{SO}_{4}\right)$ and concentrated to dryness. The resulting dipyrromethane-dicarbinol was dissolved in $\mathrm{CH}_{2} \mathrm{Cl}_{2}(168 \mathrm{~mL})$, whereupon 1d $(130 \mathrm{mg}, 0.419$ mmol) was added. After a homogeneous solution was obtained, $\mathrm{Yb}(\mathrm{OTf})_{3}$ (344 $\mathrm{mg}, 0.555$ mmol) was added. The reaction mixture was stirred at room temperature for $50 \mathrm{~min}$, and then DDQ (286 mg, $1.26 \mathrm{mmol}$ ) was added. After stirring for $1 \mathrm{~h}$, the reaction mixture was neutralized by TEA $(1 \mathrm{~mL})$ and filtered through a silica pad $\left(\mathrm{CH}_{2} \mathrm{Cl}_{2}\right)$. The purple fraction was 
collected and concentrated to dryness. The crude mixture was dissolved in $\mathrm{CHCl}_{3}(100 \mathrm{~mL})$ and the resulting solution was treated overnight with a solution of $\mathrm{Zn}(\mathrm{OAc})_{2} \cdot 2 \mathrm{H}_{2} \mathrm{O}(460 \mathrm{mg}, 2.10$ $\mathrm{mmol})$ in methanol $(25 \mathrm{~mL})$ at room temperature. The reaction mixture was washed with water, dried $\left(\mathrm{Na}_{2} \mathrm{SO}_{4}\right)$ and concentrated to dryness. The crude product was purified by column chromatography [silica, $\mathrm{CH}_{2} \mathrm{Cl}_{2}$ /hexanes (3:1)]. The resulting product was suspended in hexanes; the suspension was sonicated and then decanted to afford a purple solid (25 $\mathrm{mg}, 7 \%)$ : ${ }^{1} \mathrm{H}$ NMR $\left(\mathrm{THF}-d_{8}\right) \delta 2.52(\mathrm{~s}, 6 \mathrm{H}), 2.69(\mathrm{~s}, 3 \mathrm{H}), 4.08-4.10(\mathrm{~m}, 2 \mathrm{H}), 7.55(\mathrm{~d}, J=7.8 \mathrm{~Hz}, 4 \mathrm{H}), 7.73$ $(\mathrm{d}, J=7.8 \mathrm{~Hz}, 2 \mathrm{H}), 7.92(\mathrm{~d}, J=7.5 \mathrm{~Hz}, 2 \mathrm{H}), 8.06-8.14(\mathrm{~m}, 8 \mathrm{H}), 8.82-8.88(\mathrm{~m}, 8 \mathrm{H}) ;{ }^{13} \mathrm{C} \mathrm{NMR}$ $\left(\mathrm{THF}-d_{8}\right) \delta 21.6,29.2,30.7,119.6,121.0,121.4,121.5,121.7,122.7,127.0,127.91,127.95$, $130.4,131.8,132.1,132.19,132.23,132.3,132.5,135.3,135.5,136.9,137.7,137.8,141.6$, $141.7,141.8,141.9,143.0,143.2,143.8,150.6,151.0,151.05,151.14,151.17,151.21,151.3$; LD-MS obsd $767.2\left[(\mathrm{M}-\mathrm{Ac})^{+}\right], 809.2\left[\mathrm{M}^{+}\right] ; \lambda_{\text {abs }} 425,550,593 \mathrm{~nm} ; \lambda_{\mathrm{em}}\left(\lambda_{\mathrm{ex}}=550 \mathrm{~nm}\right) 600,648$ nm.

15-(4-(S-Acetylthiomethyl)phenyl)-10,20-di-p-tolyl-5-(4-(trifluoromethyl)phenyl)porphinatozinc(II) $\left(\mathbf{Z n P}^{\mathbf{D}} \mathbf{F}^{\mathbf{D}} \mathbf{B S A c}\right)$. Following a standard procedure ${ }^{26}$ with slight modification, ${ }^{24}$ a solution of $\mathbf{3}-\mathbf{S n B u}_{\mathbf{2}}(387 \mathrm{mg}, 0.511 \mathrm{mmol})$ in dry THF/MeOH (20 mL, 10:1) was treated with $\mathrm{NaBH}_{4}(773 \mathrm{mg}, 20.4 \mathrm{mmol})$ under argon at room temperature for $3 \mathrm{~h}$. The reaction mixture was poured into a mixture of saturated aqueous $\mathrm{NH}_{4} \mathrm{Cl}$ and $\mathrm{CH}_{2} \mathrm{Cl}_{2}$. The organic phase was separated, washed with water, dried $\left(\mathrm{Na}_{2} \mathrm{SO}_{4}\right)$ and concentrated to dryness. The resulting dipyrromethane-dicarbinol was dissolved in $\mathrm{CH}_{3} \mathrm{CN}$ (204 mL), whereupon 1 d (159 $\mathrm{mg}, 0.512 \mathrm{mmol})$ was added. After a homogeneous solution was obtained, TFA $(0.471 \mathrm{~mL}$, $0.611 \mathrm{mmol}$ ) was added. The reaction mixture was stirred at room temperature for $5 \mathrm{~min}$, then DDQ (286 mg, $1.26 \mathrm{mmol}$ ) was added. The stirring was continued for $1 \mathrm{~h}$. The reaction mixture was neutralized by TEA $(1 \mathrm{~mL})$ and filtered through a silica pad $\left(\mathrm{CH}_{2} \mathrm{Cl}_{2}\right)$. The purple fraction was collected and concentrated to dryness. The crude mixture was dissolved in $\mathrm{CHCl}_{3}(100 \mathrm{~mL})$ and the resulting solution was treated overnight with a solution of $\mathrm{Zn}(\mathrm{OAc})_{2} \cdot 2 \mathrm{H}_{2} \mathrm{O}(561 \mathrm{mg}, 2.56$ $\mathrm{mmol})$ in methanol $(25 \mathrm{~mL})$ at room temperature. The reaction mixture was washed with water, dried $\left(\mathrm{Na}_{2} \mathrm{SO}_{4}\right)$ and concentrated to dryness. The crude product was purified by column chromatography [silica, $\mathrm{CH}_{2} \mathrm{Cl}_{2}$ /hexanes (3:1)]. The resulting product was suspended in hexanes; the suspension was sonicated and then decanted to afford a purple solid (123 $\mathrm{mg}, 28 \%)$ : ${ }^{1} \mathrm{H}$ NMR $\left(\mathrm{THF}-d_{8}\right) \delta 2.46(\mathrm{~s}, 3 \mathrm{H}), 2.50(\mathrm{~s}, 3 \mathrm{H}), 2.69(\mathrm{~s}, 3 \mathrm{H}), 4.49(\mathrm{~s}, 2 \mathrm{H}), 7.56(\mathrm{~d}, J=7.8 \mathrm{~Hz}$, 4H), $7.69(\mathrm{~d}, J=7.8 \mathrm{~Hz}, 2 \mathrm{H}), 8.07(\mathrm{~d}, J=7.8 \mathrm{~Hz}, 4 \mathrm{H}), 8.09$ (d, $J=8.1 \mathrm{~Hz}, 2 \mathrm{H}), 8.12(\mathrm{~d}, J=7.8$ $\mathrm{Hz}, 2 \mathrm{H}), 8.40(\mathrm{~d}, J=8.1 \mathrm{~Hz}, 2 \mathrm{H}), 8.78(\mathrm{~d}, J=4.5 \mathrm{~Hz}, 2 \mathrm{H}), 8.82-8.89(\mathrm{~m}, 6 \mathrm{H}) ;{ }^{13} \mathrm{C}$ NMR (THF$\left.d_{8}\right) \delta 21.6,30.2,33.8,119.3,121.5,121.9,124.18,124.22,127.8,128.0,131.7,132.3,132.4$, 132.7, 135.3, 135.6, 135.8, 137.8, 138.5, 141.5, 143.3, 148.8, 150.4, 151.0, 151.2, 151.4, 194.6; LD-MS obsd $792.9\left[\left(\mathrm{M}-\mathrm{CF}_{3}\right)^{+}\right], 860.8\left[\mathrm{M}^{+}\right]$; FAB-MS obsd 860.1795, calcd 860.1775 $\left(\mathrm{C}_{50} \mathrm{H}_{35} \mathrm{~F}_{3} \mathrm{~N}_{4} \mathrm{OSZn}\right) ; \lambda_{\text {abs }} 424,550,592 \mathrm{~nm} ; \lambda_{\text {em }}\left(\lambda_{\text {ex }}=550 \mathrm{~nm}\right) 602,649 \mathrm{~nm}$.

\section{0-(4-S-Acetylthiomethylphenyl)-20-p-tolyl-5,15-bis[4-(trifluoromethyl)phenyl]-}

porphinatozinc(II) (ZnP-F ${ }^{\mathbf{L}}$ BzSAc). A solution of $1 \mathbf{c}(218 \mathrm{mg}, 0.702 \mathrm{mmol}), 1 \mathrm{~d}$ (166 mg, $0.703 \mathrm{mmol})$ and 4-(trifluoromethyl)benzaldehyde $(188 \mu \mathrm{L}, 1.41 \mathrm{mmol})$ in $\mathrm{CH}_{2} \mathrm{Cl}_{2}(140 \mathrm{~mL})$ was degassed for $5 \mathrm{~min}$ and then treated with TFA $(192 \mu \mathrm{L}, 2.49 \mathrm{mmol})$ at room temperature for $30 \mathrm{~min}$. DDQ (477 mg, $2.10 \mathrm{mmol}$ ) was added and the reaction mixture was stirred for $1 \mathrm{~h}$. After TEA $(1 \mathrm{~mL})$ was added, the reaction mixture was filtered through a silica pad $\left(\mathrm{CH}_{2} \mathrm{Cl}_{2}\right)$ and the purple fraction was collected. After removal of the solvent, the crude mixture was dissolved with $\mathrm{CHCl}_{3}(140 \mathrm{~mL})$. The resulting solution was treated overnight with a solution of $\mathrm{Zn}(\mathrm{OAc})_{2} \cdot 2 \mathrm{H}_{2} \mathrm{O}(0.771 \mathrm{~g}, 3.51 \mathrm{mmol})$ in $\mathrm{MeOH}(35 \mathrm{~mL})$ at room temperature. The reaction mixture was washed with water, dried $\left(\mathrm{Na}_{2} \mathrm{SO}_{4}\right)$ and concentrated to dryness. The resulting 
mixture of zinc porphyrins was purified by column chromatography [silica, $\mathrm{CH}_{2} \mathrm{Cl}_{2} /$ hexanes $(3: 2)]$. The desired product was suspended in hexanes; the suspension was sonicated and then decanted to afford a purple solid $(77 \mathrm{mg}, 12 \%):{ }^{1} \mathrm{H}$ NMR $\left(\mathrm{THF}-d_{8}\right) \delta 2.46(\mathrm{~s}, 3 \mathrm{H}), 2.54(\mathrm{~s}, 3 \mathrm{H})$, $4.49(\mathrm{~s}, 2 \mathrm{H}), 7.54-7.58(\mathrm{~m}, 2 \mathrm{H}), 7.66-7.71(\mathrm{~m}, 2 \mathrm{H}), 8.07$ (d, $J=7.5 \mathrm{~Hz}, 4 \mathrm{H}), 8.12$ (d, $J=7.5 \mathrm{~Hz}$, $4 \mathrm{H}), 8.40(\mathrm{~d}, J=7.8 \mathrm{~Hz}, 4 \mathrm{H}), 8.77-8.91(\mathrm{~m}, 8 \mathrm{H}) ;{ }^{13} \mathrm{C} \mathrm{NMR}\left(\mathrm{THF}-d_{8}\right) \delta 21.6,30.2,33.8,124.2$, $124.3,125.8,127.8,127.96,128.02,130.1,131.9,132.0,132.2,132.4,132.8,132.9,135.3$, 135.6, 135.8, 137.8, 138.0, 138.7, 141.4, 141.5, 143.1, 148.6, 148.8, 150.5, 150.6, 151.2, 151.4, 151.5; LD-MS obsd 806.8 [(M - 6F $\left.)^{+}\right], 860.8\left[(\mathrm{M}-3 \mathrm{~F})^{+}\right], 916.8\left[\mathrm{M}^{+}\right] ; \mathrm{M}=\mathrm{C}_{50} \mathrm{H}_{32} \mathrm{~F}_{6} \mathrm{~N}_{4} \mathrm{OSZn}$; $\lambda_{\text {abs }} 422,550,592 \mathrm{~nm} ; \lambda_{\mathrm{em}}\left(\lambda_{\mathrm{ex}}=550 \mathrm{~nm}\right) 602,648 \mathrm{~nm}$.

10-(4-S-Acetylthiomethylphenyl)-20-p-tolyl-5,15-bis[4-(trifluoromethyl)phenyl]porphyrin (P-F ${ }^{\mathbf{L}} \mathbf{B z S A c}$ ) A solution of $\mathbf{Z n P}-\mathbf{F}^{\mathbf{L}} \mathbf{B z S A c}(13 \mathrm{mg}, 14 \mu \mathrm{mol})$ in $\mathrm{CH}_{2} \mathrm{Cl}_{2}$ was treated with TFA $(1.5 \mathrm{~mL}, 19 \mathrm{mmol})$ at room temperature for $1 \mathrm{~h}$. The mixture was poured into water. The porphyrin product was extracted with $\mathrm{CH}_{2} \mathrm{Cl}_{2}$. The organic phase was dried $\left(\mathrm{Na}_{2} \mathrm{SO}_{4}\right)$, neutralized with TEA, and concentrated. The residue was chromatographed ( silica, $\mathrm{CH}_{2} \mathrm{Cl}_{2}$ ) to give the free-base porphyrin $(12 \mathrm{mg}, 99 \%):{ }^{1} \mathrm{H}$ NMR $\delta-2.83(\mathrm{~s}, 2 \mathrm{H}) 2.50(\mathrm{~s}, 3 \mathrm{H}), 2.70(\mathrm{~s}, 3 \mathrm{H})$, $4.47(\mathrm{~s}, 2 \mathrm{H}), 7.52-7.60(\mathrm{~m}, 2 \mathrm{H}), 7.63-7.71(\mathrm{~m}, 2 \mathrm{H}), 7.99-8.06(\mathrm{~m}, 4 \mathrm{H}), 8.08(\mathrm{~d}, J=7.0 \mathrm{~Hz}, 2 \mathrm{H})$, $8.13(\mathrm{~d}, J=7.7 \mathrm{~Hz}, 2 \mathrm{H}) 8.33(\mathrm{~d}, J=6.6 \mathrm{~Hz}, 4 \mathrm{H}), 8.70-8.81(\mathrm{~m}, 4 \mathrm{H}), 8.81-8.95$ (m, 4H); LD-MS obsd 799.9, 853.9; FAB-MS obsd 853.2428, calcd 853.2430 $\left(\mathrm{C}_{50} \mathrm{H}_{34} \mathrm{~F}_{6} \mathrm{~N}_{4} \mathrm{OS}\right) ; \lambda_{\text {abs }} 419,515,550$, $590,645 \mathrm{~nm}$.

\section{0-(4-S-Acetylthiomethylphenyl)-20-p-tolyl-5,15-bis[4-(trifluoromethyl)-}

phenyl]porphinatocopper(II) (CuP-F ${ }^{\mathbf{L}}$ BzSAc). A solution of P-F ${ }^{\mathbf{L}}$ BzSAc $(10 \mathrm{mg}, 12 \mu \mathrm{mol})$ in $\mathrm{CHCl}_{3}(20 \mathrm{~mL})$ was treated overnight with a solution of $\mathrm{Cu}(\mathrm{OAc})_{2} \cdot \mathrm{H}_{2} \mathrm{O}(20 \mathrm{mg}, 0.10 \mathrm{mmol})$ in $\mathrm{MeOH}(5 \mathrm{~mL})$ at room temperature. The reaction mixture was washed with water, dried $\left(\mathrm{Na}_{2} \mathrm{SO}_{4}\right)$ and concentrated to dryness. Purification by column chromatography (silica, $\mathrm{CH}_{2} \mathrm{Cl}_{2}$ ) gave a purple solid (10 mg, 93\%): LD-MS obsd 859.7, 915.7; FAB-MS obsd 913.1497, calcd $913.1522\left(\mathrm{C}_{50} \mathrm{H}_{32} \mathrm{~F}_{6} \mathrm{~N}_{4} \mathrm{OSCu}\right) ; \lambda_{\text {abs }} 415,539 \mathrm{~nm}$.

\section{0-(S-Acetylthiomethyl)-10-p-tolyl-5,15-bis[4-(trifluoromethyl)phenyl]-}

porphinatozinc(II) (ZnP-F $\left.{ }^{\mathrm{L}} \mathbf{C H}_{2} \mathbf{S A c}\right)$. Following the procedure described for $\mathbf{Z n P}-\mathbf{F}^{\mathrm{L}} \mathbf{B z S A c}$, a solution of $\mathbf{1 d}(170 \mathrm{mg}, \quad 0.720 \mathrm{mmol}), \quad \mathbf{1 e}(169 \mathrm{mg}, 0.721 \mathrm{mmol})$ and 4(trifluoromethyl)benzaldehyde $(192 \mu \mathrm{L}, 1.44 \mathrm{mmol})$ in $\mathrm{CH}_{2} \mathrm{Cl}_{2}(144 \mathrm{~mL})$ was treated with TFA (194 $\mu \mathrm{L}, 2.52 \mathrm{mmol}$ ) for $30 \mathrm{~min}$. DDQ (490 mg, $2.16 \mathrm{mmol}$ ) was added and the reaction mixture was stirred for $1 \mathrm{~h}$. After the addition of TEA $(1 \mathrm{~mL})$ and subsequent workup, the resulting crude mixture was dissolved with $\mathrm{CHCl}_{3}(140 \mathrm{~mL})$ and treated overnight with a solution of $\mathrm{Zn}(\mathrm{OAc})_{2} \cdot 2 \mathrm{H}_{2} \mathrm{O}(790 \mathrm{mg}, 3.60 \mathrm{mmol})$ in $\mathrm{MeOH}(35 \mathrm{~mL})$. After workup, the resulting mixture of zinc porphyrins was purified by column chromatography [silica, $\mathrm{CH}_{2} \mathrm{Cl}_{2} /$ hexanes $(3: 1)]$. The desired product was suspended in hexanes; the suspension was sonicated and then decanted to afford a purple solid (50 mg, 8\%): ${ }^{1} \mathrm{H}$ NMR $\left(\mathrm{THF}-d_{8}\right) \delta 2.47(\mathrm{~s}, 3 \mathrm{H}), 2.68-2.70(\mathrm{~m}$, $3 \mathrm{H}), 6.75(\mathrm{~s}, 2 \mathrm{H}), 7.56(\mathrm{dd}, J=6.9 \mathrm{~Hz}, J=4.5 \mathrm{~Hz}, 2 \mathrm{H}), 8.03-8.07(\mathrm{~m}, 2 \mathrm{H}), 8.10(\mathrm{~d}, J=7.8 \mathrm{~Hz}$, $4 \mathrm{H}), 8.38(\mathrm{~d}, J=7.8 \mathrm{~Hz}, 4 \mathrm{H}), 8.72-8.95(\mathrm{~m}, 6 \mathrm{H}), 9.56-9.67(\mathrm{~m}, 2 \mathrm{H}) ;{ }^{13} \mathrm{C}$ NMR $\left(\mathrm{THF}-d_{8}\right) \delta 21.6$, 29.8, 34.3, 114.4, 119.8, 124.2, 127.96, 128.02, 129.3, 129.7, 129.9, 131.6, 131.9, 132.4, 132.8, $132.9,133.0,135.2,135.7,137.9,138.0,141.2,141.4,148.5,150.4,150.6,151.3,151.48$, 151.52, 193.7; LD-MS obsd $655.4\left[(\mathrm{M}-\mathrm{SAc}-6 \mathrm{~F})^{+}\right], 709.2\left[(\mathrm{M}-\mathrm{SAc}-3 \mathrm{~F})^{+}\right], 763.0[[(\mathrm{M}-$ SAc $)^{+}$; FAB-MS obsd 838.1159, calcd $838.1179\left(\mathrm{C}_{44} \mathrm{H}_{28} \mathrm{~F}_{6} \mathrm{~N}_{4} \mathrm{OSZn}\right) ; \lambda_{\text {abs }}\left(\mathrm{CH}_{2} \mathrm{Cl}_{2}\right) 422,552$, $589 \mathrm{~nm}$.

15-(S-Acetylthiomethyl)-10,20-di-p-tolyl-5-(4-(trifluoromethyl)phenyl)porphinatozinc(II) $\left(\mathrm{ZnP}_{\mathbf{n}} \mathbf{F}^{\mathrm{D}} \mathbf{C H}_{2} \mathrm{SAc}\right)$. Following the procedure described for $\mathrm{ZnP}-\mathbf{D}^{\mathrm{D}} \mathbf{B z S A c}$, 


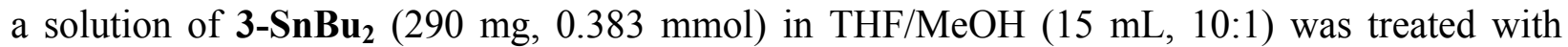
$\mathrm{NaBH}_{4}(579 \mathrm{mg}, 15.3 \mathrm{mmol})$ and worked up with a mixture of saturated aqueous $\mathrm{NH}_{4} \mathrm{Cl}(20 \mathrm{~mL})$ and $\mathrm{CH}_{2} \mathrm{Cl}_{2}(20 \mathrm{~mL})$ after $4 \mathrm{~h}$. The resulting dipyrromethane-dicarbinol was dissolved in $\mathrm{CH}_{2} \mathrm{Cl}_{2}$ $(153 \mathrm{~mL})$ and reacted with $1 \mathrm{e}(91.0 \mathrm{mg}, 0.388 \mathrm{mmol})$ in the presence of $\mathrm{Yb}(\mathrm{OTf})_{3}(313 \mathrm{mg}$, $0.505 \mathrm{mmol})$ for $45 \mathrm{~min}$. DDQ $(260 \mathrm{mg}, 1.15 \mathrm{mmol})$ was added and the stirring was continued for $1 \mathrm{~h}$. After the addition of TEA $(1 \mathrm{~mL})$ and subsequent workup, the resulting crude product was dissolved in $\mathrm{CHCl}_{3}(80 \mathrm{~mL})$ and metalated overnight with a solution of $\mathrm{Zn}(\mathrm{OAc})_{2} \cdot 2 \mathrm{H}_{2} \mathrm{O}$ (420 mg, $1.91 \mathrm{mmol})$ in $\mathrm{MeOH}(20 \mathrm{~mL})$. After workup, the crude product was purified by column chromatography [silica, $\mathrm{CH}_{2} \mathrm{Cl}_{2} /$ hexanes (3:1)]. The resulting product was suspended in hexanes; the suspension was sonicated and then decanted to afford a purple solid (94 $\mathrm{mg}, 31 \%$ ): ${ }^{1} \mathrm{H}$ NMR $\left(\right.$ THF-d $\left.d_{8}\right) \delta 2.49(\mathrm{~s}, 6 \mathrm{H}), 2.70(\mathrm{~s}, 3 \mathrm{H}), 6.75(\mathrm{~s}, 2 \mathrm{H}), 7.56(\mathrm{~d}, J=7.8 \mathrm{~Hz}, 4 \mathrm{H}), 8.06(\mathrm{~d}, J$ $=7.8 \mathrm{~Hz}, 4 \mathrm{H}), 8.07(\mathrm{~d}, J=7.8 \mathrm{~Hz}, 2 \mathrm{H}), 8.36(\mathrm{~d}, J=7.8 \mathrm{~Hz}, 2 \mathrm{H}), 8.73(\mathrm{~d}, J=4.8 \mathrm{~Hz}, 2 \mathrm{H}), 8.83$ $(\mathrm{d}, J=4.8 \mathrm{~Hz}, 2 \mathrm{H}), 8.92(\mathrm{~d}, J=4.8 \mathrm{~Hz}, 2 \mathrm{H}), 9.60(\mathrm{~d}, J=4.8 \mathrm{~Hz}, 2 \mathrm{H}) ;{ }^{13} \mathrm{C} \mathrm{NMR}\left(\mathrm{THF}-d_{8}\right) \delta$ 21.6, 29.8, 34.4, 114.1, 119.7, 121.9, 124.2, 127.9, 129.4, 131.7, 132.7, 133.3, 135.3, 135.7, 137.9, 141.4, 148.7, 150.5, 151.3, 193.7; LD-MS obsd 709.6 [(M - SAc $\left.)^{+}\right]$; FAB-MS obsd 784.1495, calcd 784.1462 $\left(\mathrm{C}_{44} \mathrm{H}_{31} \mathrm{~F}_{3} \mathrm{~N}_{4} \mathrm{OSZn}\right) ; \lambda_{\text {abs }}\left(\mathrm{CH}_{2} \mathrm{Cl}_{2}\right) 421,552,591$.

\section{0,15,20-Tri-p-tolyl-5-[4-(trifluoromethyl)phenyl]porphinatozinc(II) $\quad$ (ZnP-CF 3 ).} Following the procedure described for $\mathbf{Z n P}-\mathbf{D}^{\mathbf{D}} \mathbf{B z S A c}$, a solution of $\mathbf{4}$ (122 $\left.\mathrm{mg}, 0.258 \mathrm{mmol}\right)$ in THF/MeOH (10 mL, 10:1) was treated with $\mathrm{NaBH}_{4}(195 \mathrm{mg}, 5.16 \mathrm{mmol})$ for $40 \mathrm{~min}$. Workup with a mixture of saturated aqueous $\mathrm{NH}_{4} \mathrm{Cl}(20 \mathrm{~mL})$ and $\mathrm{CH}_{2} \mathrm{Cl}_{2}(20 \mathrm{~mL})$ gave the dipyrromethane-dicarbinol. The latter was dissolved in $\mathrm{CH}_{2} \mathrm{Cl}_{2}(100 \mathrm{~mL})$ and reacted with $\mathbf{1 b}$ $(75.0 \mathrm{mg}, 0.258 \mathrm{mmol})$ in the presence of $\mathrm{Yb}(\mathrm{OTf})_{3}(205 \mathrm{mg}, 0.331 \mathrm{mmol})$ for $40 \mathrm{~min}$. DDQ (176 $\mathrm{mg}, 0.775 \mathrm{mmol}$ ) was added and the stirring was continued for $1 \mathrm{~h}$. After the addition of TEA ( $1 \mathrm{~mL})$ and subsequent workup, the resulting crude product was dissolved in $\mathrm{CHCl}_{3}(70$ $\mathrm{mL})$ and metalated overnight with a solution of $\mathrm{Zn}(\mathrm{OAc})_{2} \cdot 2 \mathrm{H}_{2} \mathrm{O}(283 \mathrm{mg}, 1.29 \mathrm{mmol})$ in $\mathrm{MeOH}$ $(15 \mathrm{~mL})$. After workup, the crude product was purified by column chromatography [silica, $\mathrm{CH}_{2} \mathrm{Cl}_{2}$ /hexanes (3:1)]. The resulting product was suspended in hexanes; the suspension was sonicated and then decanted to afford a purple solid $(52 \mathrm{mg}, 26 \%):{ }^{1} \mathrm{H}$ NMR $\left(\mathrm{THF}-d_{8}\right) \delta 2.70(\mathrm{~s}$, 9H), 7.56 (d, $J=7.8 \mathrm{~Hz}, 6 \mathrm{H}), 8.06$ (d, $J=7.8 \mathrm{~Hz}, 6 \mathrm{H}), 8.08-8.10(\mathrm{~m}, 2 \mathrm{H}), 8.39$ (d, $J=7.8 \mathrm{~Hz}$, 2H), $8.77(\mathrm{~d}, J=4.8 \mathrm{~Hz}, 2 \mathrm{H}), 8.83-8.88(\mathrm{~m}, 6 \mathrm{H}) ;{ }^{13} \mathrm{C}$ NMR $\left(\mathrm{THF}-d_{8}\right) \delta 21.6,30.7,119.1,121.8$, 122.0, 124.2, 128.0, 131.6, 132.1, 132.3, 132.4, 132.6, 135.3, 135.7, 137.8, 141.6, 148.9, 151.4, 151.16, 151.24, 151.4; LD-MS obsd 732.5 [(M - 3F $\left.)^{+}\right], 786.5\left[\mathrm{M}^{+}\right]$; FAB-MS obsd 786.1959, calcd $786.1949\left(\mathrm{C}_{48} \mathrm{H}_{33} \mathrm{~F}_{3} \mathrm{~N}_{4} \mathrm{Zn}\right) ; \lambda_{\text {abs }}\left(\mathrm{CH}_{2} \mathrm{Cl}_{2}\right) 420,548,581 \mathrm{~nm}$.

5-[4-(4-Allylhepta-1,6-dien-4-yl)phenyl]-15-[4-(trifluoromethyl)phenyl]-10,20-di-ptolylporphinatozinc(II) $\left(\mathbf{Z n P}-\mathbf{F}^{\mathbf{D}} \mathbf{T}\right.$ pd). Following a standard procedure, ${ }^{24,26}$ a solution of tin complex 5-SnBu $(100 \mathrm{mg}, 0.121 \mathrm{mmol})$ in $\mathrm{THF} / \mathrm{MeOH}(20 \mathrm{~mL}, 10: 1)$ was treated with $\mathrm{NaBH}_{4}$ $(227 \mathrm{mg}, 6.00 \mathrm{mmol}$ ) in small portions with rapid stirring at room temperature. After $4 \mathrm{~h}$, the reaction was quenched by slow addition of saturated aqueous $\mathrm{NH}_{4} \mathrm{Cl}$. The reaction mixture was extracted with $\mathrm{CH}_{2} \mathrm{Cl}_{2}$. The organic solution was dried $\left(\mathrm{Na}_{2} \mathrm{SO}_{4}\right)$ and concentrated, affording 5diol as a slightly yellow foam-like solid. The freshly prepared 5-diol was condensed with 1f (35.1 mg, $0.121 \mathrm{mmol})$ in $\mathrm{CH}_{2} \mathrm{Cl}_{2}(48 \mathrm{~mL})$ containing $\mathrm{Yb}(\mathrm{OTf})_{3}(95.1 \mathrm{mg}, 3.2 \mathrm{mM}, 0.153 \mathrm{mmol})$ at room temperature for $35 \mathrm{~min}$. DDQ $(90 \mathrm{mg}, 0.40 \mathrm{mmol})$ was added and the reaction mixture was stirred for $1 \mathrm{~h}$. TEA was added. The reaction mixture was filtered through a pad of silica $\left(\mathrm{CH}_{2} \mathrm{Cl}_{2}\right)$. The first fraction was collected and concentrated. The purple solid was dissolved in $\mathrm{CHCl}_{3}(20 \mathrm{~mL})$ and a solution of $\mathrm{Zn}(\mathrm{OAc})_{2} \cdot 2 \mathrm{H}_{2} \mathrm{O}(200 \mathrm{mg}, 0.911 \mathrm{mmol})$ in methanol $(6 \mathrm{~mL})$ was added. The reaction mixture was stirred overnight at room temperature. Chromatography (silica, $\mathrm{CH}_{2} \mathrm{Cl}_{2}$ ) afforded a purple solid (19 mg, 17\%): ${ }^{1} \mathrm{H} \mathrm{NMR}(400 \mathrm{MHz}) \delta 2.72(\mathrm{~s}, 6 \mathrm{H}), 2.74$ 
$(\mathrm{d}, J=7.6 \mathrm{~Hz}, 6 \mathrm{H}), 5.18-5.23(\mathrm{~m}, 6 \mathrm{H}), 5.84-5.94(\mathrm{~m}, 3 \mathrm{H}), 7.56(\mathrm{~d}, J=7.3 \mathrm{~Hz}, 4 \mathrm{H}), 7.69(\mathrm{~d}, J=$ $7.3 \mathrm{~Hz}, 2 \mathrm{H}), 8.03$ (d, $J=7.6 \mathrm{~Hz}, 2 \mathrm{H}), 8.11(\mathrm{~d}, J=7.3 \mathrm{~Hz}, 4 \mathrm{H}), 8.17$ (d, $J=8.0 \mathrm{~Hz}, 2 \mathrm{H}), 8.35$ (d, $J=7.6 \mathrm{~Hz}, 2 \mathrm{H}), 8.86(\mathrm{~d}, J=4.6 \mathrm{~Hz}, 2 \mathrm{H}), 8.94(\mathrm{~d}, J=4.6 \mathrm{~Hz}, 2 \mathrm{H}), 8.96-9.01(\mathrm{~m}, 4 \mathrm{H}) ;{ }^{13} \mathrm{C} \mathrm{NMR}$ $(100 \mathrm{MHz}) \delta 21.8,29.9,42.3,44.0,118.2,121.7,123.8,125.2,127.6,131.5,132.3,132.4,132.6$, 134.4, 134.6, 134.7, 134.9, 137.4, 139.9, 140.3, 145.3, 149.8, 150.6, 150.8; LD-MS obsd 906.4; FAB-MS obsd 906.2896, calcd $906.2888\left(\mathrm{C}_{57} \mathrm{H}_{45} \mathrm{~F}_{3} \mathrm{~N}_{4} \mathrm{Zn}\right) ; \lambda_{\text {abs }}\left(\mathrm{CH}_{2} \mathrm{Cl}_{2}\right) 421,549,588, \mathrm{~nm}$.

Dibutyl[5,10-dihydro-1,9-bis[4-(trifluoromethyl)benzoyl]-5-p-tolyl]dipyrrinato]tin(IV) (6-SnBu $\left.\mathbf{~}_{2}\right)$ Following a standard procedure, ${ }^{28} \mathrm{MesMgBr}(8.5 \mathrm{~mL}, 8.5 \mathrm{mmol}, 1.0 \mathrm{M}$ in THF) was added slowly to a tap-water cooled flask containing a solution of $\mathbf{1 d}(400 \mathrm{mg}, 1.69$ $\mathrm{mmol})$ in THF $(20 \mathrm{~mL})$ under argon. The reaction mixture was stirred at room temperature for $30 \mathrm{~min}$. A sample of 4-(trifluoromethyl)benzoyl chloride $(0.629 \mathrm{~mL}, 4.23 \mathrm{mmol})$ was added over $10 \mathrm{~min}$. The mixture was stirred for $1 \mathrm{~h}$ and then was poured into a mixture of saturated aqueous $\mathrm{NH}_{4} \mathrm{Cl}$ and ethyl acetate. The organic layer was washed (water and brine), dried $\left(\mathrm{Na}_{2} \mathrm{SO}_{4}\right)$, and concentrated to dryness. The residue was treated with TEA (1 mL) and $\mathrm{Bu}_{2} \mathrm{SnCl}_{2}$ (513.5 mg, $1.69 \mathrm{mmol})$ in $\mathrm{CH}_{2} \mathrm{Cl}_{2}(20 \mathrm{~mL})$. The mixture was stirred at room temperature for 30 min and then concentrated. Chromatography [silica, $\mathrm{CH}_{2} \mathrm{Cl}_{2} /$ hexanes (1:1)] afforded a yellow solid (560 mg; 41\%): mp 55-58 ${ }^{\circ} \mathrm{C}$ (dec.); ${ }^{1} \mathrm{H}$ NMR $(400 \mathrm{MHz}) \delta 0.70(\mathrm{t}, J=7.0 \mathrm{~Hz}, 3 \mathrm{H}), 0.76(\mathrm{t}$, $J=7.0 \mathrm{~Hz}, 3 \mathrm{H}), 1.13-1.54(\mathrm{~m}, 10 \mathrm{H}), 1.67-1.72(\mathrm{~m}, 2 \mathrm{H}), 2.31(\mathrm{~s}, 3 \mathrm{H}), 5.57(\mathrm{~s}, 1 \mathrm{H}), 6.22(\mathrm{~d}, J=$ $4.1 \mathrm{~Hz}, 2 \mathrm{H}), 7.05(\mathrm{~d}, J=3.8 \mathrm{~Hz}, 2 \mathrm{H}), 7.10(\mathrm{~s}, 4 \mathrm{H}), 7.75(\mathrm{~d}, J=8.3 \mathrm{~Hz}, 4 \mathrm{H}), 7.98(\mathrm{~d}, J=8.0 \mathrm{~Hz}$, $4 \mathrm{H}) ;{ }^{13} \mathrm{C}$ NMR $(100 \mathrm{MHz}) \delta 13.8,21.2,24.3,25.0,26.2,26.5,27.5,45.5,116.2,124.8,126.6$, $128.1,129.5,129.7,135.7,136.8,140.9,153.2$.

\section{5-[4-(4-Allylhepta-1,6-dien-4-yl)phenyl]-15-p-tolyl-10,20-bis[4-(trifluoromethyl)-} phenylporphinatozinc(II) $\left(\mathbf{Z n P}-\mathbf{F}^{\mathbf{L}} \mathbf{T p d}\right)$ Following standard procedures, ${ }^{24,26,28,30}$ a solution of tin complex 6-SnBu $(110 \mathrm{mg}, 0.136 \mathrm{mmol})$ in THF $(20 \mathrm{~mL})$ was treated with $\mathrm{NaBH}_{4}(52 \mathrm{mg}$, $1.37 \mathrm{mmol})$. A sample of methanol $(7 \mathrm{~mL})$ was then added dropwise by syringe over $10 \mathrm{~min}$. After $45 \mathrm{~min}$, TLC (silica, $\mathrm{CH}_{2} \mathrm{Cl}_{2}$ ) indicated incomplete reduction. Therefore, an additional quantity of $\mathrm{NaBH}_{4}\left(52 \mathrm{mg}, 1.37 \mathrm{mmol}\right.$ ) was added. After $0.5 \mathrm{~h}$, TLC (silica, $\mathrm{CH}_{2} \mathrm{Cl}_{2}$ ) showed complete reduction. The reaction mixture was quenched by slow addition of saturated aqueous $\mathrm{NH}_{4} \mathrm{Cl}$. The reaction mixture was extracted with $\mathrm{CH}_{2} \mathrm{Cl}_{2}$. The organic solution was dried $\left(\mathrm{Na}_{2} \mathrm{SO}_{4}\right)$ and concentrated, affording 6-diol as a slightly yellow foam-like solid. The freshly prepared 6-diol was condensed with 1 f $(48.3 \mathrm{mg}, 0.136 \mathrm{mmol})$ in $\mathrm{CH}_{2} \mathrm{Cl}_{2}$ (54.4 mL) containing $\mathrm{Yb}(\mathrm{OTf})_{3}(108 \mathrm{mg}, 3.2 \mathrm{mM}, 0.174 \mathrm{mmol})$ at room temperature for $35 \mathrm{~min}$. DDQ $(93 \mathrm{mg}, 0.41$ mmol) was added. TEA was added, and the reaction mixture was filtered through a pad of silica $\left(\mathrm{CH}_{2} \mathrm{Cl}_{2}\right)$. The first fraction was collected and concentrated. The purple solid was dissolved in $\mathrm{CHCl}_{3}(20 \mathrm{~mL})$, and a solution of $\mathrm{Zn}(\mathrm{OAc})_{2} \cdot 2 \mathrm{H}_{2} \mathrm{O}(300 \mathrm{mg}, 1.37 \mathrm{mmol})$ in methanol $(6 \mathrm{~mL})$ was added. The reaction mixture was stirred overnight at room temperature. After workup and concentration to dryness, the crude product was purified by column chromatography (silica, $\mathrm{CH}_{2} \mathrm{Cl}_{2}$ ). The resulting product was suspended in hexanes; the suspension was sonicated and then decanted to afford a purple solid (44 mg, 34\%): ${ }^{1} \mathrm{H}$ NMR $(400 \mathrm{MHz}) \delta 2.72-2.75(\mathrm{~m}, 9 \mathrm{H})$, $5.18-5.23(\mathrm{~m}, 6 \mathrm{H}), 5.82-5.95(\mathrm{~m}, 3 \mathrm{H}), 7.57(\mathrm{~d}, J=7.6 \mathrm{~Hz}, 2 \mathrm{H}), 7.69(\mathrm{~d}, J=8.3 \mathrm{~Hz}, 2 \mathrm{H}), 8.03$ $(\mathrm{d}, J=8.0 \mathrm{~Hz}, 4 \mathrm{H}), 8.10(\mathrm{~d}, J=8.0 \mathrm{~Hz}, 2 \mathrm{H}), 8.16(\mathrm{~d}, J=8.3 \mathrm{~Hz}, 2 \mathrm{H}), 8.36(\mathrm{~d}, J=7.6 \mathrm{~Hz}, 4 \mathrm{H})$, 8.87-8.90 (m, 4H), $8.97(\mathrm{~d}, J=4.5 \mathrm{~Hz}, 2 \mathrm{H}), 9.02(\mathrm{~d}, J=4.5 \mathrm{~Hz}, 2 \mathrm{H}) ;{ }^{13} \mathrm{C} \mathrm{NMR}(100 \mathrm{MHz}) \delta$ $21.8,42.3,44.0,118.2,119.6,123.8,125.2$, 127.6, 131.8, 131.8, 132.7, 132.8, 134.4, 134.6, 134.7, 134.8, 137.6, 139.7, 140.0, 145.5, 146.8, 149.9, 150.7, 150.8; LD-MS obsd 960.5; FABMS obsd 960.2617, calcd 960.2605 $\left(\mathrm{C}_{57} \mathrm{H}_{42} \mathrm{~F}_{6} \mathrm{~N}_{4} \mathrm{Zn}\right) ; \lambda_{\text {abs }}\left(\mathrm{CH}_{2} \mathrm{Cl}_{2}\right) 421,548,586 \mathrm{~nm}$. 